\title{
The Flocs Target Detection Algorithm Based on the Three Frame Difference and Enhanced Method of the Otsu
}

\author{
Xin Xie, Huiping Li, and Fengping Hu
}

\begin{abstract}
At present, the commonly used floc tracking algorithms are susceptible to noise, light, and floc movement speed, etc., it is difficult to extract complete moving flocs, therefore put forward a flocs target detection algorithm based on the three frame difference and particle swarm optimization \& enhanced method of the Otsu. The algorithm firstly conducts difference of three consecutive frames, then use particle swarm optimization \& enhanced method of the Otsu to obtain the best threshold, the optimal threshold is adopted to the image binarization and then do image post-processing, eventually obtaining a clear floc target, to lay a foundation for subsequent automation analysis of floc. Experiments show that the algorithm has the characteristics of fast, accurate, and can extract flocs target effectively.
\end{abstract}

Index Terms-Three frame difference, particle swarm optimization \& enhanced method of the Otsu, optimal threshold, flocs target detection.

\section{INTRODUCTION}

As people living standard unceasing enhancement, the people's requirements to the drinking water quality also more and more strict, conventional water treatment process generally consists of coagulation, sedimentation, filtration, disinfection and so on several stages at home and abroad .

Condensation is the phenomenon occurring during the tap water processin, so the number, size and settling velocity of flocs are important parameters to determine the coagulation effect. Moving target detection is an important step in quantitative analysis of flocs image, also affect the flocs analysis automation, work stability and accuracy of results. due to the problems exist in the process of imaging, like non-uniform illumination, slow movement speed, changes of floc distribution, etc., the image obtained by industrial camera appear some phenomenon like the flocs of color shades, poor uniformity, at the same time, there is noise and there are surface different reflective of floc, So to achieve the detection of flocs and background accurately, is hard, but is the basis of further analyzing the characteristic of flocs [1].

There are many methods used for the detection of flocs targets, like the background difference method [2], frame difference method [3] and optical flow method.

Frame difference method is 2 or 3 adjacent frames in the video sequence, using per-pixel difference and threshold to extract the target in the image, it can rapidly detect the

Manuscript received March 11, 2014; revised May 15, 2014

Xin Xie and Huiping Li are with the East China Jiaotong University of information Engineering College, Nanchang, China (e-mail: xienew@gmail.com, 812584127@qq.com).

Fengping $\mathrm{Hu}$ is with School of Civil Engineering, ECJT University, Nanchang, Jiangxi, China (e-mail: hufegnping1968@126.com). changed part of the video image. Frame difference method is simple, better adaptability to the environment and have high stability, Three frame difference method is the improvement of second frame difference, but as floc target movement speed is slow, due to the threshold selection is inappropriate, such as bimodal method, can lead to a uncomplete extraction of floc target information, even for some slow speed flocs will occur leak phenomenon, therefore, aimed at the current problems of frame differential method, and threshold selection method, this paper proposes a flocs target detection algorithm based on the three frame difference and particle swarm optimization $\&$ enhanced method of the Otsu.

\section{BASIC PRINCIPLE OF IMAGE PROCESSING}

\section{A. Two Frames Difference Method}

Two frames difference method is the most simple and most direct method in motion target detection, through the difference between two frames can quickly detect the moving targets. To get image sequence of a certain period of time in the continuous image sequence, set $f_{t}(x, y)$ as the current frame of t time, set $f_{t+1}(x, y)$ as a frame after the $t$ time, set $M_{t}(x, y)$ as moving target, as pixel in the background, $n_{t}(x, y)$ as noise.the two frame difference method as follows [4] :

1) Assuming that the outside light conditions did not change or changed a little, the representation of adjacent image sequence is as follows:

$$
\begin{aligned}
& f_{t}(x, y)=M_{t}(x, y)+B_{t}(x, y)+n_{t}(x, y) \\
& f_{t+1}(x, y)=M_{t+1}(x+\bar{x}, y+\bar{y})+B_{t+1}(x, y)+n_{t+1}(x, y)
\end{aligned}
$$

2) Get two adjacent frame difference image from subtracting the two formula:

$$
\begin{aligned}
& Q_{\text {diff }}(x, y)=f_{t+1}(x, y)-f_{t}(x, y)=M_{t+1}(x+\bar{x}, y+\bar{y})- \\
& M_{t}(x, y)+B_{t+1}(x, y)-B_{t}(x, y)+n_{t+1}(x, y)-n_{t}(x, y)
\end{aligned}
$$

3) Binarization

$$
R_{t}(x, y)=\left\{\begin{array}{l}
1, \text { if }\left(Q_{\text {diff }}(x, y) \geq T\right) \\
0, \text { if }\left(Q_{\text {diff }}(x, y)<T\right)
\end{array}\right.
$$

$$
M_{t+1}(x+\bar{x}, y+\bar{y})-M_{t}(x, y) \text { is the change of image }
$$
caused by the moving object, $B_{t+1}(x, y)-B_{t}(x, y)$ is the 
background difference between adjacent frames, $n_{t+1}(x, y)-n_{t}(x, y)$ is the noise between adjacent frames, $\mathrm{T}$ for the threshold.

Using (3) to calculate the image difference between the $t$ frame and $t+1$ frame, calculation results is difference image $Q_{\text {diff }}(x, y)$, according to binarization algorithm to select appropriate threshold, and use it to do the difference image binarization, so as to obtain relevant information of moving targets, then according to actual circumstances, using image processing technology to process subsequent process.

\section{B. Three Frame Difference Method}

Because of the area isolated by two frames difference method tend to be larger than the actual, often will appear the phenomenon of "double", and three frame difference method has greater improvement on the basis of this, Calculate $f_{t-1}(x, y)$ with $f_{t}(x, y)$ to get difference image $D_{t}, f_{\mathrm{t}+1}(x, y)$ with $f_{t}(x, y)$ to get the difference image $D_{t+1}$, then, let $D_{t+1}$ and $D_{t}$ do "\&" operation to get difference image $Q_{\text {diff }}(x, y)$, finally choosing optimal threshold,For three frame difference method, selecting reasonable threshold plays an important role for object extraction, difference image thresholding is achieved according to (4) to get binary results [4].The basic flow of three frame difference method is shown in Fig. 1:

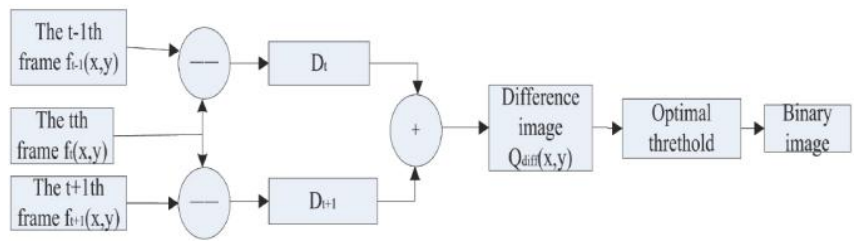

Fig. 1. Principle of three frame differencing.

\section{The Otsu}

According to the different ways to choose threshold value, basically have bimodal method, iterative method, the between-cluster variance method (the Otsu). Although the bimodal method is simple, but it has poor adaptability; the computation of iterative method is huge, is not suitable for real-time system; the Otsu method works best relatively. The Otsu method firstly, let the image pixel be divided into two kinds through threshold $\mathrm{T}$, then calculate infra -class and inter-class variance between two classes of pixel gray value, for the maximum ratio between the two, to determine the threshold T [4]-[6].

But in simulation experiment, we found that, when deal with the situation that there is not much gray level difference between the background and prospect target, using the Otsu will appear a lot of black areas, seriously even will lose the target. Some scholars put forward into a gray stretch to enhance the Otsu method, namely using nonlinear transform to do Grey-scale extension of image, and then determine the threshold value, but it also increases the operation time.

\section{The Particle Swarm Optimization Algorithm (PSO)}

Particle swarm optimization algorithm (PSO) is a kind of optimization algorithm that imitate such behaviors in the nature based on iterative. In PSO, the solution of optimization problem can be seen as a "particles", each particle has two basic attributes that are: a position $x$ and velocity $\mathrm{v}$, the particle's position and speed as shown in (5) and (6):

$$
\begin{gathered}
x_{i}=\left(x_{i 1}, x_{i 2}, x_{i 3}, \ldots, x_{i n}\right) \\
v_{i}=\left(v_{i 1}, v_{i 2}, v_{i 3}, \ldots, v_{i n}\right)
\end{gathered}
$$

In the formula, $n$ is problem's dimension, PSO is initialized to a group of random particles, the process of algorithm is to calculate each particle's fitness function, and then particles update themselves by tracking two extremum: one is the current optimal position, known as " $p_{\text {best }}$ ", the other one is the optimal location of current particle within the neighborhood, known as" $n_{\text {best }}$ ",when finding the two optimal, particle update themselves according to (7) and (8)

$$
\begin{gathered}
v=w v+c_{1} r_{1}\left(p_{\text {best }}-x\right)+c_{2} r_{2}\left(n_{\text {best }}-x\right) \\
x=x+v \Delta t
\end{gathered}
$$

In the formula, $w$ is inertia weight, $c_{1}, c_{2}$ are the study factor, $r_{1}, r_{2}$ are two separate random number that satisfy uniform distribution, scope between $(0,1) . \Delta t$ is Time intervals.

\section{IMPROVED ALGORITHM}

\section{A. Particle Swarm Optimization \& Enhanced Method of the Otsu}

Enhance method of the Otsu improves the effect of the Otsu method due to the introduction of gray stretch, but also increase the operation time, when applied to real-time operation, the threshold value can't be chosen timely, As for the demand of time, this article improves the Enhance method of the Otsu, which uses the particle swarm optimization to optimize Enhance method of the Otsu, First of all give a random solution to the particle's position and speed, because the range of a gray image is between 0-255, so we give a position to the particle in the range, all the random value generated by the rand () function. Then calculate the value of fitness function and comparison, then do the iterative calculation, finally obtaining the optimal solution, which is the best threshold.

During the calculation process, selection of Weight $w$ and $c_{1}, c_{2}$ parameters is very important, and the choice of weight affect the searching ability of the algorithm. Usually, we hope it have stronger global search ability in the first time, later, focus on the enhancement of local search ability; Generally, in the process of search values of $w$ change from 0.9 to 0.4 in a linear gradient way, in order to make the particles find the optimal value more efficient, generally take $c_{1}=c_{2}=2$.

\section{B. Three Frame Difference and Particle Swarm Optimization \& Enhanced Method}

Comparison with two frame difference method, the three 
frame difference method is used to solve the problem of sensitivity to the target movement speed in a certain extent, but for slowly moving target, if choose an inappropriate threshold value will cause the bad effect ; If the threshold is too large, will cause the missing of target; if the threshold value is too small, will make too much noise, So according to the features of three frame difference and the motion characteristics of flocs, propose a flocs target detection algorithm based on the three frame difference and particle swarm optimization \& enhanced method of the Otsu, namely three frame difference method is used to detect floc target, at the same time, in the process of the algorithm, using the particle swarm optimization \& enhanced method of the Otsu to obtain the best threshold.

Detailed process of the algorithm is as follows:

1) Let the current frame $I_{t}$ and the previous frame $I_{t-1}$, and the later frame $I_{t+1}$ do the smoothly denoising respectively;

2) And, get $I_{t-1}, I_{t}, I_{t+1}$ do the difference respectively, and get the difference image $D_{t+1}, D_{t}$;

3) The difference image do the "\&" manipulations to get the difference image $D_{i}$;

4) Using the improved the Otsu method that is particle swarm optimization \& enhanced method of the Otsu to determine the optimal threshold value;

5) Difference image binarization is realized according to formula (4) and the best threshold, and finally determine the binary image ;

6) Using post-processing technology of image processing to deal with the image in (5) step, such as: inflation, etc., so that make the incomplete boundary complete, and the boundary of the complete image is obtained, thereby achieve the goal that extract flocs target smoothly.

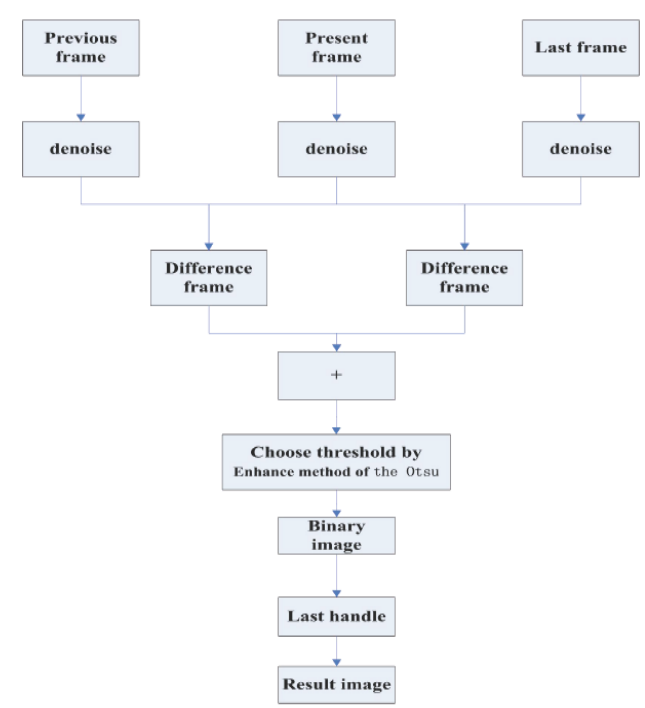

Fig. 2. The flowchart of the improved algorithm.

\section{ANALYSIS OF EXPERIMENTAL RESULTS}

Install sensors in the water at the end of the flocculation pool, and make water flow through sampling window slowly, then collect water (floc) images of sampling window by industrial camera continuously, In the experiment, we set the sampling time interval according to the actual demand. In this experiment the time interval Tsampe is $1 \mathrm{~s}$. (within $1 \mathrm{~s}$ time, flocs in the observation form have very good representative), particle number is around 100, can be used as sample to process images. On the platform of PC, we can achieve the processing of the image sequence through $\mathrm{VC}++6.0$ and OpenCV1.0 by the proposed method. Test results as shown in figure 3 .

Fig. 3 (a) - (c) is three images extracted for every 5 frames (in order to observe, extract the same area of the three images randomly;

Fig. 3 (d) - (f) is the result of the two frames difference method; Fig. $3(\mathrm{~g})$ - (I) is the result for the three frame method combined with the iteration method; Figure 3 (j) (l) is the result for the three frame method combined with of the Otsu method; Fig. 3 (m) - (o) is the results of the improved algorithm proposed in this paper. We can see from the result of the experiment: when using the two frame difference algorithm for moving object ,the extraction is not very complete, appeared the phenomenon of a lot of empty area; Using iteration method with three frame difference algorithm, when the difference of target and background region area is larger, the images detection effect is poorer, and lost a lot of information of the image at the time of the flocs image detection, some small flocs which gray close to background cannot be detected; the Otsu method with three frame difference algorithm, when the difference between target background and prospect in gray level is not big, it will appear a black area, seriously even will lose the target. From the experimental results in this paper, we can see that the improved algorithm can extract the edges of the moving flocs more accurately, the extraction of flocs target is more complete, especially for larger area of target, the extraction effect is good, solved some problems that exist in the traditional three frame difference algorithm to a certain extent, like the edge detection is not complete, the shortcomings of appear target "empty" phenomenon, can achieve flocs extraction of the target area accurately and timely.

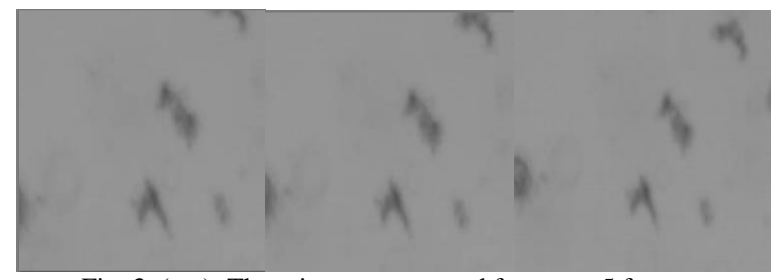

Fig. 3. (a-c): Three images extracted for every 5 frames.

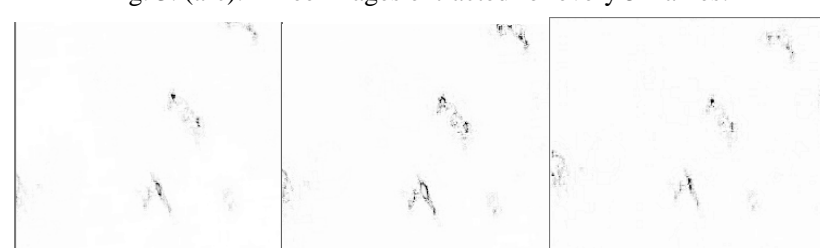

Fig. 3. (d-f): The result of the two frames difference method.

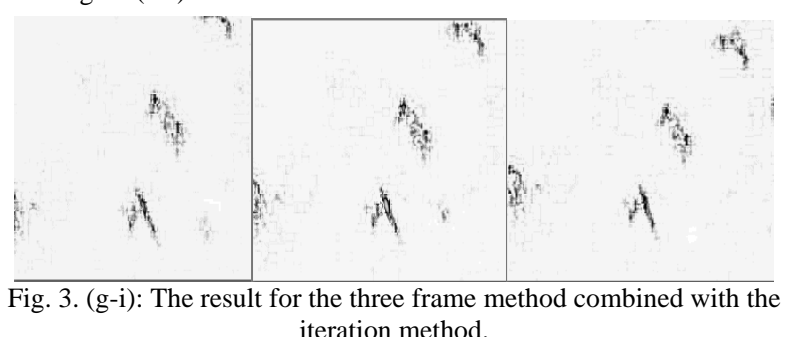




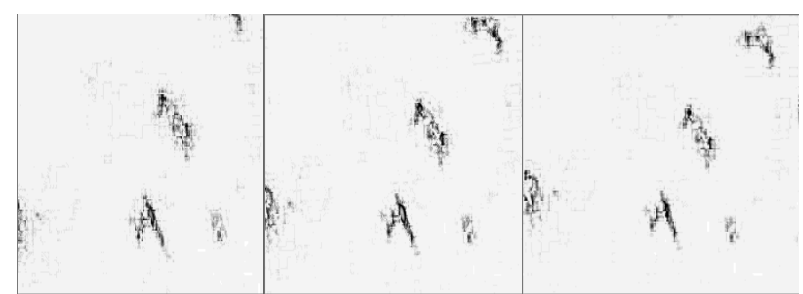

Fig. 3. (j-1): The result for the three frame method combined with of the Otsu method.

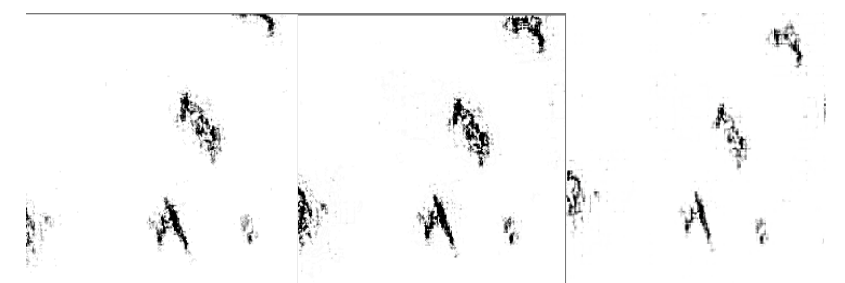

Fig. 3. (m-o): The results of the improved algorithm proposed in this paper.

\section{CONCLUSION}

An improved algorithm is proposed in this paper, that is a flocs target detection algorithm based on the three frame difference and particle swarm optimization \& enhanced method of the Otsu. The improved algorithm adopts the three frame difference algorithm to extract the flocs target, avoid the possible problems that using the two frame difference algorithm extracts target such as incomplete extraction, but at the same time in three frame difference algorithm, it exists the problems of the optimum threshold selection, this paper uses particle swarm optimization \& enhanced method of the Otsu to select the best threshold, effectively solved the problem of threshold selection is not reasonable and low efficiency when adopting the method of iteration, As a result, the proposed algorithm has the advantages like fast, accurate, extract the flocs target completely, better antinoise performance, the experimental results show that the method has certain practical value, can extract target floc effectively. But in the case of relatively small flocs target, the effect is not obvious by using the method to extract, it needs to be improved at next step.

\section{ACKNOWLEDGMENT}

This work is supported by National Natural Science Foundation of China under Grant No. 61272197, and by Innovation Special Funds Projects for Graduate Students of ECJT University in Jiangxi Province under Grant No.YC2012-X017, and by Natural Science Foundation of Jiangxi Province under Grant No.20132BAB201027.

\section{REFERENCE}

[1] Z. Li, "Research on in-situ idenification technology of floc morphology in water," Harbin Institute of Technology, Harbin, pp. 350, 2008.

[2] H. Hartaohui and L. Davis, "Real-time surveillance of people and their activities." IEEE Trans Pattern Analysis and Machine Intelligence, vol. 22, no. 8, pp. 809-830, 2000.

[3] D. Meyer, L. J. Denz, and H. N. Iemann, "Model based extraction of articulated objects in image sequences for gait analysis," in Proc IEEE Interl national Conference on Image Processing, Santa Barbara, California,1997, pp. 78-81

[4] H.-J. Zhou and Y.-L. Zhou, "Study on bubble segmentation method based on particle swarm optimization \& enhanced method of the Otsu," Journal of Northeast Dianli University, vol. 31, no. 1, pp. 6976, 2011.

[5] A. Lipton, H. Fujiyoshi, and R. Patil, "Moving target classification and tracking from real-time video," in Proc. IEEE Workshop on Application of Computer Vision, Princeton: NJ, pp. 8-14, 1998.

[6] G. Rui, "Research on improved algorithm of detection and tracking based on three frame difference and camshift," Yanshan University, pp. 25-42, 2011.

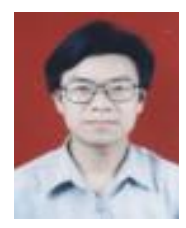

Xin Xie received his master's degree from Nanchang University of Control Theory and Control Engineering in Nanchang, he is a professor in the East China JiaoTong University of Information Engineering College. His research interests are machine vision, computer software.

Huiping $\mathbf{~ L i}$ is studying in East China Jiaotong University of Information Engineering Institute, and now is a graduate student. Huiping Li's main research directions are machine vision and image processing.

Fengping Hu received his Ph.D. from Chongqing University of Environmental Engineering in Chongqing, China. He is a professor in the East China Jiaotong University of Civil Engineering and Construction. His research interest is environmental engineering. 